\title{
Effect of intervention on the use of Metered Dose Inhaler amongst the children suffering with asthma and their caregivers
}

\author{
Veena Ranaut*, Sukhwinder Kaur**, Sukhpal kaur**, Meenu Singh*** \\ *Clinical Instructor, Sister Nivedita Govt. College, IGMC Shimla (Himachal Pradesh) \\ **Lecturer, NINE, PGIMER, Chandigarh. \\ ***Professor, Deptt. of Pediatrics, PGIMER, Chandigarh.
}

\begin{abstract}
:
Background: Asthma is one of the most common worldwide chronic conditions and is considered a major health problem in children. Limited knowledge and poor management skills regarding use of MDI identified as the major reasons for the high morbidity and mortality.

Objective: To develop skill in use of MDI among the children suffering with asthma and their caregivers.

Design: Operational study

Setting: Asthma clinic, Advanced Pediatrics Centre, PGIMER, Chandigarh.

Participants/Patients: By using Purposive sampling technique 100 diagnosed children with bronchial asthma between age group of 3-14 yrs were enrolled in the study. The tools used for data collection were semistructured interview schedule to gather the information regarding socio-demographic profile of the subjects, knowledge, performance and practice of using the MDI. A protocol regarding right technique of using inhaler was developed in Hindi and English. An observation checklist was used to observe the steps/ technique of using MDI by the subjects.

Material and Methods: Each subject was interviewed personally. After assessing the baseline performance scores, the right technique of using MDI was demonstrated. The demonstrations were repeated until the children and caregivers developed proficiency. Power point presentation was also prepared and showed to all subjects. Booklet was given to each subject. All the subjects were asked to come for the follow up after fifteen days interval. Skill of using MDI was assessed in three subsequently follow up visits by using follow up data sheet. Maximum attainable score was 14 for using inhaler without spacer \& 15 score for using inhaler with spacer.
\end{abstract}

Result: Mean age of the subjects was $7.6 \pm 0.6$ yrs with the range of 3-14 yrs. 56\% subjects were in the age group of 6-10 yrs. Out of total subjects, $86 \%$ were using inhaler. Out of them, $3.4 \%$ achieved very low scores, $65.3 \%$ were at low scores and 31.3\% were attained average scores. Whereas 14 subjects were using inhaler without spacer. Out of them, 92.9\% were at low and $7.1 \%$ subjects attained average score. None of the subject in both the groups was at the good or excellent score. Baseline mean performance score of all the subjects was $6.81 \pm 1.1$ which increased to $11.39 \pm 1.03,12.60 \pm 1.14$ and $13.98 \pm .09$ subsequently in first, second and third follow up respectively.

Conclusion: Repeated demonstrations regarding use of MDI are required to make the person skillful and proficient.

Key words: Patients, Skill, MDI

\section{Introduction}

Bronchial asthma is one the most common diseases of childhood. Around $4-20 \%$ school going children in India suffer from asthma.$^{1-2}$

Treatment of asthma by inhalation devices is in practice since many years. MDIs are often preferred inhalation devices. Combined with a spacer device it is more convenient especially in children and elderly people. The purpose of the spacer device is to act as an intermediary chamber into which the MDI can discharge the drug allowing to inhale over several breaths. A secondary advantage of using spacers with inhaledcorticosteroid is the incidence of local side effects, such as oropharyngeal candidiasis and hoarseness, is reduced. ${ }^{3}$

In order to achieve asthma control it is essential that children receive appropriate education and training pertaining to the management of their disease and most importantly, are prescribed the correct inhalation device to ensure that medication is deposited in their lungs. ${ }^{3}$ However it has been observed that patients have lack of skill in using MDI.

Many asthma-affected children and their parents are not familiar with appropriate techniques for inhaler use. This may result in misuse, overdose, or diminished response of the administered therapeutic drugs, or may even result in unnecessary, repeated hospitalization. ${ }^{3}$ 
A study by Khassawneh et al ${ }^{4}$ revealed that more than half of the subjects were not able to perform all the steps of using the inhaler, only $30 \%$ could reach the total possible scores of nine.

The selection of an inhaler device and training the patient in its correct use may be delegated explicitly or implicitly. A substantial proportion of patients may receive no inhaler instruction. A study on 'primary practice audit of asthma management' was conducted in Canada, revealed that $28 \%$ of 6,684 patients reported never having demonstration on their inhaler technique. 221 Canadian physicians who participated in the same practice audit estimated that $25 \%$ of their patients had never demonstrated their inhaler technique ${ }^{5}$ This Review focuses on the needs of demonstration of right technique of using MDI.

Numerous studies have identified sub-optimal inhaler technique as a common problem in patients with respiratory disease. ${ }^{6-7}$ Early studies clearly showed that patients struggled with conventional MDIs. Although many errors in handling were detected, the most common was difficulty coordinating device actuation and inhalation. ${ }^{8}$ Investigators working in referral centres, such as pulmonary function laboratories, have estimated that between $14-89 \%$ of patients seen for the first time will use their inhalers sub-optimally. ${ }^{7-9}$

However, if these studies are examined critically, it becomes clear that not all handling errors are crucial. For example, although failure to remove the cap from a MDI before use will clearly render it useless, failing to shake the device before the second dose of inhalations may have little effect on drug delivery.

It has been demonstrated that a significant proportion of patients do not shake the device properly or present with coordination problem, resulting in variable amounts of drug emitted not reaching to target site (respiratory system). The occurrence of mistakes associated with the inhalation procedures can be reduced by regular training and follow up. Patient education has shown a significant impact on the performance scores of the subjects. Yoon et al ${ }^{10}$ found a significant improvement in knowledge in mean scores from $2.89 \pm 1.02$ (baseline) to $3.09 \pm 0.91$ at 10 months follow ups. A study was conducted in Taiwan ${ }^{3}$ to evaluate the skills needed for inhaler use among children with asthma. It is revealed that $17 \%$ of the subjects had good compliance with medical treatment but none of subject was observed to complete all the steps of the inhaler technique correctly. It was concluded that patients were not taking medication through inhaler properly. Hence majority of patients are deprived of maximum benefits from inhaler therapy.

It has been opined that to obtain maximum impact, asthma education needs to be relevant, realistic and repeated. ${ }^{11}$ Instructions in the more practical aspects of self monitoring of asthma and regular use of preventive medication are required. Teaching the patient how to use MDIs correctly is not sufficient. Reinforcing the inhalation technique is of utmost importance.

The current study was carried out to observe the effect of an intervention and regular enforcement regarding use of MDI amongst the children suffering with asthma and their caregivers.

\section{Material and methods}

The study was conducted in the asthma clinic Advanced Pediatric Centre, Post Graduate Institute of Medical education and Research, Chandigarh. Using purposive sampling technique, 100 diagnosed children with bronchial asthma between age group of 3-14 yrs and their care givers who accompany the child were enrolled in the study. Diagnosis of child was confirmed from OPD card written by physician.

The interview schedule was prepared after reviewing the relevant literature and under the guidance of experts from the Departments of Pediatric Pulmonology and nursing education. It consisted of five parts. Information was obtained regarding demographic profile of children and their caregivers. Multiple choice questions were prepared to assess the knowledge of subjects regarding asthma and its management. A checklist consisted of 14 items for using inhaler without spacer and 15 items for using inhaler with spacer to assess baseline performance score. There were maximum 14 score for using inhaler without spacer \& 15 scores for using inhaler with spacer.

The protocol was prepared in the form of pictorial booklet under the headings like definition, etiology, signs and symptoms, precipitating factors, goal of treatment, commonly used drugs, household management of mild, moderate and severe episodes of asthma. All the subsequent steps regarding use of inhalers with or without spacer were described in booklet and power point presentations were also made to enhance learning skills.

Each subject was asked to demonstrate the use of inhaler with or without spacer. Each item was scored for correct response. Score 1 was given to the correct response and 0 to incorrect or missed step. Subjects were asked to give return demonstrations. The demonstrations were repeated until children and caregivers developed proficiency in using MDI. Each subject was asked to come for the follow up. The subjects who could not come for the first follow up were contacted telephonically and motivated for visit. The subjects who achieved excellent scores in first follow up were not called for second follow up. Performance was categorized as 'very low', 'low', 'average', 'good' and ' excellent' as per score obtained by the subjects. Content validity of the questionnaire was established by giving the tool amongst the respective faculties in National Institute of Nursing Education and experts in the field of Pulmonology. 
Ethical approval for the study was obtained from the Ethical Institution Committee. There was no interference on the routine practices of the subjects while collecting data. Professional norms were maintained. Informed consent was obtained from each subject. Descriptive and inferential statistics was used to summarize the data, and group differences were analyzed by chi-square for categorical variables and by ANOVA for continuous variables.

\section{Identification data of the children}

\section{Results}

Majority (97\%) were old treated cases. $27 \%$ of subjects were less than 5 years of age, $56 \%$ were between 5 to 10 years of age and seventeen were between 10-15 years of age. The mean age of the subjects was $7.7 \pm 0.6$ with the range of 3-14 years. Around three fourth (73\%) subjects were male. All 100 subjects were studying in school. $46 \%$ were studying between nursery to $3^{\text {rd }}$ standard and remaining $54 \%$ subjects were studying between $4^{\text {th }}$ to $8^{\text {th }}$ standard. $76 \%$ subjects were suffering with asthma since 1-5 years and 21 subjects were suffering for more than 5 years.

Table 1: Identification data of the children

$\mathbf{N}=\mathbf{1 0 0}$

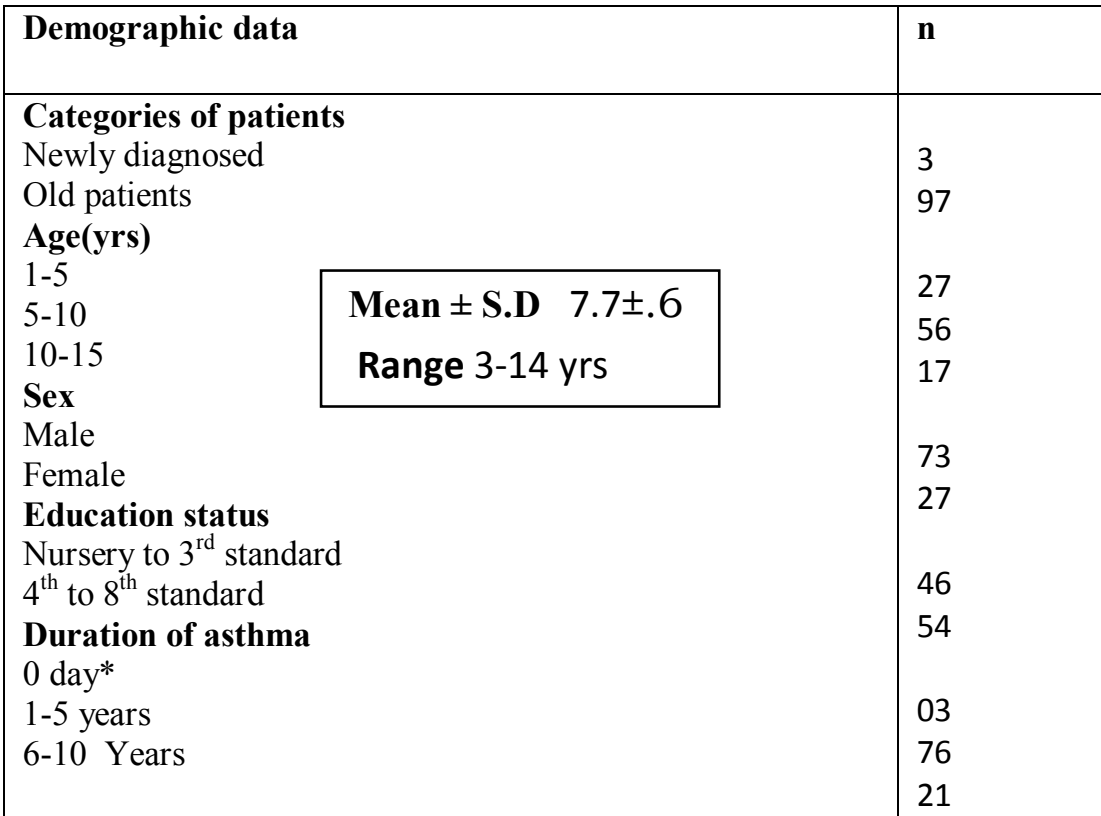

\section{Identification data of the caregivers}

Table 2 depicts that $90 \%$ of caregivers were in the age group of $24-45$ years. Mean age of the caregivers was $36.2 \pm 1.9$ years with the range of $24-64$ years. $28 \%$ were under-matric, $64 \%$ were undergraduates, and only $8 \%$ subjects were postgraduate. $42 \%$ mothers were home-maker where as $32 \%$ were professionals. $44 \%$ fathers were professionals. Monthly income of around one third $(37 \%)$ of the caregivers was ranging from

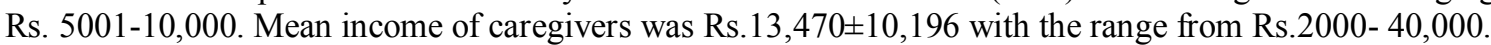

Table 2: Socio-demographic profile of care givers.

\section{$\mathbf{N}=\mathbf{1 0 0}$}




\begin{tabular}{|c|c|c|}
\hline \multicolumn{2}{|c|}{ Demographic data of care givers } & \multirow[b]{3}{*}{55} \\
\hline Age(yrs) & & \\
\hline $24-35$ & Mean \pm S.D $6.2 \pm 1.9$ & \\
\hline $\begin{array}{l}36-45 \\
>15\end{array}$ & Range 24-64 yrs & 35 \\
\hline \multicolumn{3}{|l|}{ Sex } \\
\hline Male & & 52 \\
\hline Female & & 48 \\
\hline \multicolumn{3}{|c|}{ Education status } \\
\hline Under matr & & 28 \\
\hline undergradu & & 64 \\
\hline Post gradua & & 08 \\
\hline \multicolumn{3}{|c|}{ Occupation of mother } \\
\hline Home mak & & 42 \\
\hline Professiona & & 32 \\
\hline \multirow{2}{*}{\multicolumn{3}{|c|}{ Occupation of father }} \\
\hline & & \\
\hline Unemploye & & 07 \\
\hline Professiona & & 44 \\
\hline Retired, sk & cultivator & 49 \\
\hline \multicolumn{3}{|c|}{ Monthly income of family (in Rs.) } \\
\hline$\leq 5000$ & & 24 \\
\hline $5001-10,00$ & & 37 \\
\hline $10001-2000$ & & 23 \\
\hline $20001-3000$ & & 08 \\
\hline$>30000$ & & 08 \\
\hline \multicolumn{3}{|c|}{ Per capita income (in Rs.) } \\
\hline$\leq 500-1500$ & & 53 \\
\hline $1501-3000$ & & 23 \\
\hline $3001-5000$ & & 13 \\
\hline$>5000$ & & 11 \\
\hline
\end{tabular}

Baseline performance scores of the subjects using inhaler with or without spacer

Figure-1 depicts the baseline assessment scores of total 100 subjects. 86 subjects were using inhaler with spacer where as 14 were using inhalers without spacer. Out of 86 subjects, $3(3.4 \%)$ subjects scored very low (0-3), $56(65.3 \%)$ were at low (4-7) scores and 27(31.3\%) attained average (8-11) scores. None of the subject in both the groups was at the good or excellent scores.

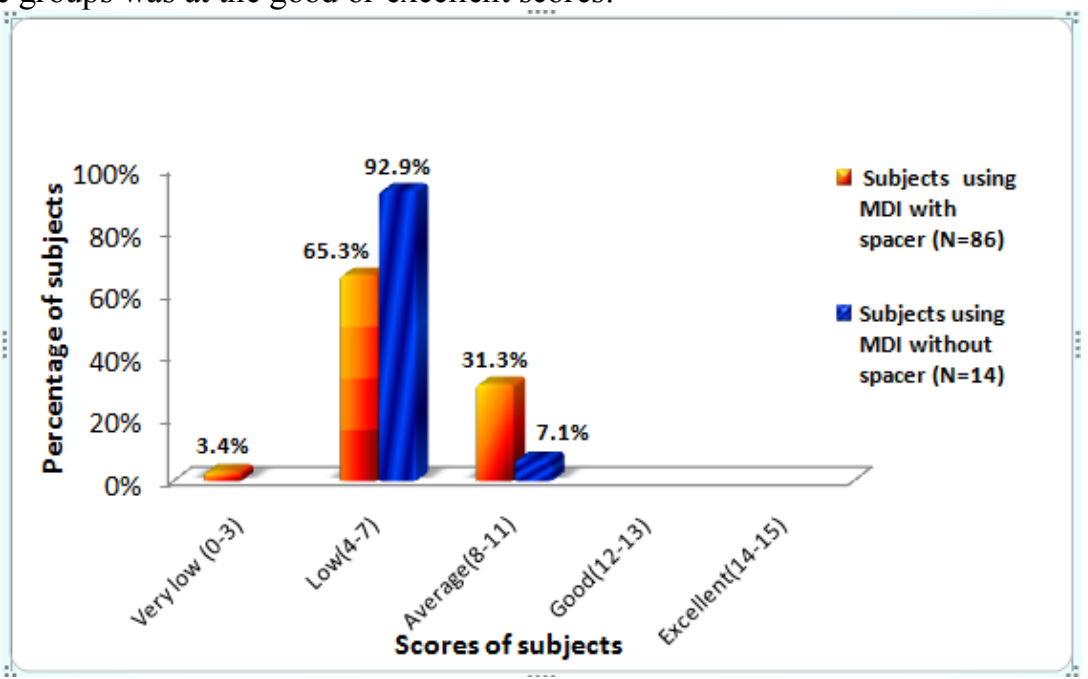

Figure-1 Baseline performance scores of the subjects regarding use of inhaler Number of demonstrations required to achieve proficiency regarding use of inhaler with or without spacer. 
Maximum four demonstrations were required to learn all the steps correctly for the subjects using inhaler without spacer. $21.4 \%$ subjects learnt after $2^{\text {nd }}$ demonstration; $57.1 \%$ after $3^{\text {rd }}$ demonstration and $28.6 \%$ after $4^{\text {th }}$ demonstration.

Total six demonstrations were required to learn all the steps correctly for the subjects using inhaler with spacer. $15 \%$ subjects achieved maximum score after $2^{\text {nd }}$ demonstration; $55.8 \%$ subjects after $3^{\text {rd }}$ demonstration; $23 \%$ after $4^{\text {th }}$ demonstration and $4 \%$ after $5^{\text {th }}$ demonstration (fig 2 ).

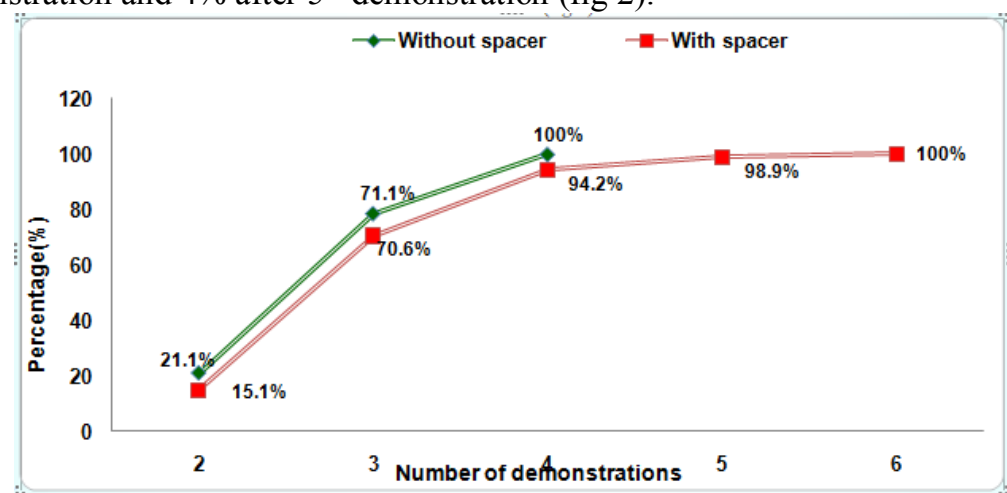

Figure-2 Number of demonstrations required to achieve proficiency

Performance score of the subjects using inhaler with spacer during follow-up visits (item wise)

Table 3 depicts the item wise response of the subjects using inhaler with spacer. In all the steps of using inhaler there was increase in the number of subjects on each successive follow up visit. Out of total subjects, $1.2 \%$ ensured right prescribed dose in the baseline assessment. After the demonstration, $44.3 \%, 63.8 \%$ and $80.4 \%$ subjects were able to perform this step in each follow up. It was found that hand washing was performed by $6.1 \%$ in the baseline assessment, and this number increased to $39.5 \% ; 65.2 \%$ and $74.5 \%$ in the 1 st, $2^{\text {nd }}$ and $3^{\text {rd }}$ follow up respectively. $48.7 \%$ subjects were able to shake their inhaler before use in the baseline assessment where as the percentage of subjects increased $88.6 \%, 92.8 \%$ and $96.1 \%$ in $1^{\text {st }}, 2^{\text {nd }}$ and $3^{\text {rd }}$ follow up respectively.

It was observed that only $4.8 \%$ subjects practiced test firing in the baseline assessment. The number of subjects increased up to $57 \%, 72.4 \%$ and $80.4 \%$ in each follow up. $35.6 \%$ subjects were exhaling before taking MDI in the baseline assessment whereas $60.5 \%, 81.2 \%$ and $83.3 \%$ subjects were able to breathe out before using inhaler in $1^{\text {st }}, 2^{\text {nd }}$ and $3^{\text {rd }}$ follow up respectively. In the baseline assessment, it revealed that $9.8 \%$ subjects tilted their head before taking the inhaler but number of subjects increased up to $89.9 \%$ in first and second follow up and $96.1 \%$ in third follow up. Regarding rinsing the mouth after using inhaler it was found that only $2.4 \%, 62 \%, 82.6 \%$ and $92.2 \%$ subjects were practiced the same step in the baseline, $1^{\text {st }}, 2^{\text {nd }}$ and $3^{\text {rd }}$ follow up respectively.

Table3: Performance score of the subjects using inhaler with spacer during follow-up visits (itemwise)

\begin{tabular}{|c|c|c|c|c|c|}
\hline $\begin{array}{l}\text { Sr. } \\
\text { no. }\end{array}$ & Steps of using MDI with spacer & $\begin{array}{c}\text { Baseline } \\
\mathbf{n}=86 \\
\mathbf{n}(\%)\end{array}$ & $\begin{array}{cl}1^{\text {st }} & \text { followup } \\
& \mathbf{n}=79 \\
& \mathbf{n}(\%)\end{array}$ & $\begin{array}{c}2^{\text {nd }} \text { Followup } n=69 \\
n(\%)\end{array}$ & $\begin{array}{c}\mathbf{3}^{\text {rd }} \text { followup } \\
\mathbf{n = 5 1} \\
\mathbf{n}(\%)\end{array}$ \\
\hline 1 & Ensures the right prescribed dose. & $1(1.2)$ & $35(44.3)$ & $44(63.8)$ & $41(80.4)$ \\
\hline 2 & Checks label \& expiry date of medicine. & $1(1.2)$ & $31(39.5)$ & $43(43.3)$ & $44(86.3)$ \\
\hline 3 & Washes hands before use. & $5(6.1)$ & $31(39.5)$ & $45(65.2)$ & $38(74.5)$ \\
\hline 4 & Shakes well before use. & $40(48.7)$ & $70(88.6)$ & 64(92.8) & $49(96.1)$ \\
\hline 5 & Takes off the cover from the mouth piece & $86(100)$ & $79(100)$ & $69(100)$ & $51(100)$ \\
\hline 6 & Tests fire by releasing one puff in the air & $4(4.8)$ & $45(57)$ & $50(72.4)$ & $41(80.4)$ \\
\hline 7 & $\begin{array}{l}\text { Assembles spacer by fitting the two parts } \\
\text { together }\end{array}$ & $80(97.6)$ & $79(100)$ & $69(100)$ & $51(100)$ \\
\hline 8 & Breathes out slowly before dosing & $30(35.6)$ & $48(60.5)$ & $56(81.2)$ & $43(83.3)$ \\
\hline 9 & Tilts head slightly back. & $8(9.8)$ & $71(89.9)$ & $62(89.9)$ & $49(96.1)$ \\
\hline 10 & Places mouth piece of spacer \& form a & $80(97.6)$ & $79(100)$ & $69(100)$ & $51(100)$ \\
\hline
\end{tabular}


Effect of intervention on the use of Metered Dose Inhaler amongst the children suffering with asthma

\begin{tabular}{|c|l|l|l|l|l|}
\hline & tight seal & & & \\
\hline 11 & $\begin{array}{l}\text { Puts one puff in holding chamber of } \\
\text { spacer \& start inhaling slowly }\end{array}$ & $79(96.3)$ & $79(100)$ & $69(100)$ & $51(100)$ \\
\hline 12 & $\begin{array}{l}\text { Removes the spacer \& holds the breath } \\
\text { for 5 to 10 seconds. }\end{array}$ & $67(81.7)$ & $62(78.5)$ & $55(79.7)$ & $49(96.1)$ \\
\hline 13 & $\begin{array}{l}\text { Breathes out slowly. Repeat 8-13 steps } \\
\text { for 2 dose }\end{array}$ & $80(97.6)$ & $78(98.7)$ & $69(100)$ & $51(100)$ \\
\hline 14 & $\begin{array}{l}\text { Rinses mouth with water } \\
15\end{array}$ & $\begin{array}{l}\text { Cleans the mouth piece of inhaler \& } \\
\text { spacer with water }\end{array}$ & $12(14.6)$ & $68(86.1)$ & $57(82.6)$ \\
\hline
\end{tabular}

Performance score regarding use of MDI as per the level of score achieved by the subjects

Before the demonstration, more than half $(65.3 \%)$ of the subjects using inhaler with spacer were at low score and $31.3 \%$ were at average score. In the $1^{\text {st }}$ follow up, out of 79 subjects, $45.5 \%$ were at good score. $3.7 \%$ were at excellent score. In the $2^{\text {nd }}$ follow up, $10 \%$ subjects were at average scores, around three- fourth $(76.8 \%)$ subjects achieved good scores and 9 (13\%) were at excellent scores. In the 3rd follow up, one third (33.3\%) subjects achieved good scores and around three-fourth of the subjects $(66.3 \%)$ were at the excellent scores.

92.9\% subjects using inhaler without spacer achieved low scores (4-7) in the baseline assessment. In the $1^{\text {st }}$ follow up, out of total 12 subjects, nearly half $(41.6 \%)$ were at good scores. $8.4 \%$ were at excellent scores. In the 2nd follow up, nearly three-fourth 70\% subjects achieved good scores and 2(20\%) were at excellent scores. In the 3rd follow up visit, majority of the subjects $87.5 \%$ were at excellent scores(table 4, fig 3 )

Table 4: Performance score regarding use of MDI as per the level of score achieved by the subjects

$\mathbf{N}=86$

Max. $\operatorname{scores}=15$

\begin{tabular}{|c|c|c|c|c|c|c|c|c|}
\hline Inhalers & Follow up visits & $\mathbf{N}$ & $\begin{array}{l}\text { Very } \\
\text { low } \\
(0-3) \\
\end{array}$ & $\begin{array}{l}\text { Low } \\
(4-7)\end{array}$ & $\begin{array}{c}\text { Average } \\
(8-11)\end{array}$ & $\begin{array}{l}\text { Good } \\
(12-13)\end{array}$ & $\begin{array}{c}\text { Excellent } \\
(14-15)\end{array}$ & $\begin{array}{c}\text { F } \\
\text { P-value }\end{array}$ \\
\hline \multirow[t]{4}{*}{$\begin{array}{l}\text { With } \\
\text { spacer }\end{array}$} & $\begin{array}{l}\text { Baseline } \\
\text { assessment }\end{array}$ & 86 & $3(3.4)$ & $56(65.5)$ & $27(31.3)$ & ---- & ---- & \multirow[t]{4}{*}{$\begin{array}{c}424.041 \\
0.000 * * *\end{array}$} \\
\hline & $1^{\text {st }}$ follow up & 79 & ---- & ------ & $40(50.6)$ & $37(45.5)$ & $3(3.7)$ & \\
\hline & $2^{\text {nd }}$ follow up & 69 & ------ & ------ & $7(10.2)$ & $53(76.8)$ & $9(13.0)$ & \\
\hline & $3^{\text {rd }}$ follow up & 51 & ---- & ---- & & $17(33.3)$ & $34(66.3)$ & \\
\hline \multirow[t]{4}{*}{$\begin{array}{l}\text { Without } \\
\text { spacer }\end{array}$} & $\begin{array}{l}\text { Baseline } \\
\text { assessment }\end{array}$ & 14 & ---- & 13(92.9) & $1(7.1)$ & ------ & ----- & \multirow[t]{4}{*}{$\begin{array}{l}121.967 \\
.000 * * *\end{array}$} \\
\hline & $1^{\text {st }}$ follow up & 12 & ------ & ------ & $6(50)$ & $5(41.6)$ & $1(8.4)$ & \\
\hline & $2^{\text {nd }}$ follow up & 10 & ---- & ---- & $1(10)$ & $7(70)$ & $2(20)$ & \\
\hline & $3^{\text {rd }}$ follow up & 8 & ---- & ----- & ----- & $1(12.5)$ & $7(87.5)$ & \\
\hline
\end{tabular}

Mean scores regarding use of inhaler in each sucessive

follow up visits

$\rightarrow$ With spacer $\rightarrow$-Without spacer

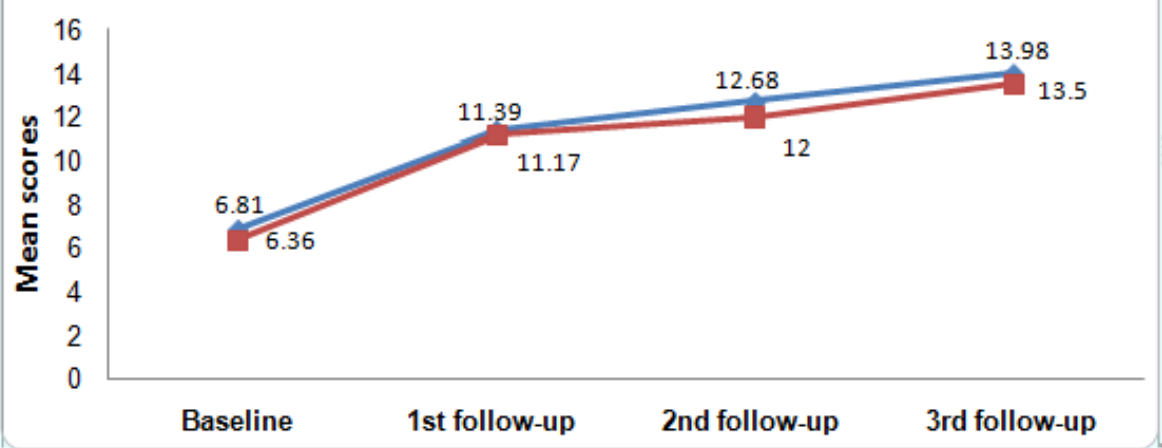

Figure-3 Mean scores in each successive follow up visit

There was a significant difference among the mean scores of baseline assessment, $1^{\text {st }}, 2^{\text {nd }}$ and $3^{\text {rd }}$ follow up of subjects using inhaler with spacer. $(\mathrm{F}=424.01, \mathrm{p}=0.00)$ and without spacer $(\mathrm{F}=121.967, \mathrm{p}=.000)(\operatorname{table} 4$, fig 3) 


\section{Discussion}

Medical treatment and care provided to asthmatic patients may not help them much until the right techniques of using inhaler are demonstrated. Many studies have shown that providing written materials may help patients to recall information. ${ }^{3}$ But repeated demonstrations from the subjects are equally important to check their immediate memory and regular enforcement through follow ups to make learning skills more concrete and consistent. There are various ways of educating children but demonstration is preferred.

In the current study, repeated demonstrations were given to the patients and their caregivers to make them proficient in using MDI. $86 \%$ of the subjects were using MDI with spacer while $14 \%$ were using it without the spacer. Though the subjects were using inhalers since years, yet none of them could achieve good or excellent scores in the baseline assessment. Khassawneh et al ${ }^{4}$ conducted a study on 'assessment of inhalation technique' in college of pharmacy, King Saudi University amongst the medical personnel. The result of his study showed that most the patient were using incorrect technique. Another study conducted in Taiwan, revealed that though $17 \%$ of the subjects had good compliance with medical treatment but none of the subject was observed to complete all the steps of the inhaler technique correctly.

Another study conducted by Paterson and Crompton ${ }^{12}$ found that despite instructions by medical personnel in use of the inhaler 45 out of 321 asthmatic subjects still did not use the MDI's as instructed.

Several studies have focused on the effects of demonstration on people's ability to use inhalers. Heringa et al ${ }^{13}$ considered both the knowledge and psychomotor skills of the patients and concluded that a structured teaching of right technique is an effective method for improving skills regarding the use of the inhaler with or without spacer. He concluded that only one individual was using the MDI with the right techniques (i.e. scored 16/16) on the baseline assessment. Repeated demonstrations were given to improve their performance scores. In the present study also five or six demonstrations were given to make the subjects proficient.

In the current study, out of 86 subjects who were using the inhaler with a spacer, $79 \%$ came for first follow up. In the first follow up, half of the subjects achieved average score whereas $45.5 \%$ attained good scores. 69 subjects came in the $2^{\text {nd }}$ follow up. $1 / 3^{\text {rd }}$ achieved good scores where as $13 \%$ attained excellent scores. In the $3^{\text {rd }}$ follow up 59 subjects were followed. Out of them, $33.3 \%$ were at good scores whereas $2 / 3^{\text {rd }}$ was at excellent scores. The mean performance scores of the subjects regarding use of the MDI were significantly higher in all three follow ups visits as compare to baseline. Yoon et al ${ }^{10}$ found in his study a significant improvement in performance scores from $2.89 \pm 1.02$ to $3.09 \pm 0.91$ at 10 months follow ups. In current study, baseline performance mean score increased from $6.81 \pm 1.1$ to $11.39 \pm 1.6$ on interval of fifteen days. Though the time interval was less as compared to results of Yoon et al ${ }^{10}$ yet remarkable increase in mean scores were found. Similar findings have been reported by Wilson et al. ${ }^{14}$

In the present study, there was a marked increase in the number of subjects giving the correct response at each subsequent follow-up. In the $1^{\text {st }}$ follow up, $80 \%$ of the subjects were shaking the inhaler before using it. Only half of the subjects were test firing while purchasing a new inhaler and more than half were able to exhaled breath before using inhaler. Maximum subjects had tilted their head back while taking inhaler. Only less than half were rinsing their mouth after using the inhaler. More than half of the subjects were cleaning the mouth of the spacer daily with plain water.

The results of the current study showed a definite advantage of conducting follow up visits as the performance scores of subjects were improved in each successive follow up. It shows that the patients improved their knowledge by repeatedly consulting booklets as well as by personal interactions with health personnel. Similar results are reported by Bauman ${ }^{15}$ where the knowledge of the patients regarding common asthma medication (inhaled steroids and inhaled and oral bronchodilators) was assessed. The overall proportion of correct responses increased by following the self management programme.

There is need to operationalize such protocols, continue reinforcement and supplements asthma management programme at short interval. Thus it will help to improve the quality of life of asthmatic children and reduced asthma mortality and morbidity. It has been opined that to obtain maximum impact, asthma education needs to be relevant, realistic and repeated.

\section{Conclusion}

It is important that one should take inhalation medications regularly but utmost important that the technique to use inhaler should be proper. Repeated demonstrations and re-demonstrations regarding use of inhaler help the subjects with bronchial asthma to improve their performance scores. Study findings have revealed that knowledge and performance scores of subjects improved significantly after teaching right technique of using inhalers. 


\section{References}

[1]. Lieu TA, Lozano P, Finkelstein JA et al. Racial/ethnic variation in asthma status and management practices among children in managed Medicaid. Pediatrcis 2002; 109; 857-65.

[2]. Awasthi S, Kalra E, Roy S. Prevalence and risk factors of asthma and wheeze in school-going children in Lucknow, North India. Indian Pediatr 2004; 41: 1205-10.

[3]. Sue-Hsien Chen SH, Yin TJC, Huang JL. An exploration of the skills needed for inhalation therapy in school children with asthma in Taiwan. Annals of Allergy, Asthma and Immonology 2002; 89(3): 311-15.

[4]. Khassawneh BFY, MK AL Ali, K H Alzeudi, MZ Batarex, HM Alnasr. Handling out of inhaler devices in acual pulmonary practice MDI vs dry powder inhaler. Respaire. Care 2008;53: 314-15

[5]. Chapman KR, Boulet LP, Rea R. Physician self-audit of asthma control in primary practice: the implications for implementation of guidelines. Am J Respir Crit Care Med 2004; 169: A362

[6]. Hesselink AE, Penninx BW, Wijnhoven HA, Kriegsman DM, van Eijk JT. Determinants of an incorrect inhalation technique in patients with asthma or COPD. Scand J Prim Health Care 2001; 19: 255-260.

[7]. Coady TJ, Davies HJ, Barnes P. Evaluation of a breath actuated pressurized aerosol. Clin Allergy 1976; 6: 1-6.

[8]. Epstein SW, Manning CP, Ashley MJ, Corey PN. Survey of the clinical use of pressurized aerosol inhalers. Can Med Assoc J 1979; 120: 813-816.

[9]. Coady TJ, Stewart CJ, Davies HJ. Synchronization of bronchodilator release. Practitioner 1976; 217: $273-275$.

[10]. Yoon R, Mckenzie D, Bauman A and Miles D. Controlled trial of an asthma education through computer supported education for asthma patients. BMJ 1993; 308: 568-71

[11]. Kolbe J,Vamos M, Janes S, Elkind, G and Garett J. Assessment of practical knowledge of self manangement of acute asthma. Chest1996; 109:89-90.

[12]. Paterson IC, Crompton GK. Use of pressurised aerosols by asthmatic patients. BMJ 1976; 1: 76-77.

[13]. Heringa, P, Lawson L and Reda d. The effect of structured education program on the knowledge and psychomotor skills of the patients using beclomethasone dipropionate aerosol for steroid dependent asthma. Health educ. Q 1987;14: 309-17

[14]. Wilson SR, Scamagas P and German DF. A control trails of two forms of self management education for adultwith asthma AJM. 1993; 94: 564-76

[15]. Brauman A, Mitchell CA, Henry RL. Asthma morbidity in Australia: an epidemiological study. AJM. 1992; 156:827-31 